\title{
Iterative Detection of Diagonal Block Space Time Trellis Codes, TCM and Reversible Variable Length Codes for Transmission over Rayleigh Fading Channels
}

\author{
S. X. Ng, F. Guo and L. Hanzo
}

\author{
School of ECS, University of Southampton, SO17 1BJ, UK. \\ Tel: +44-23-8059 3125, Fax: +44-23-8059 4508 \\ Email: $\{$ sxn,fg01r,lh\}@ecs.soton.ac.uk, http://www-mobile.ecs.soton.ac.uk
}

\begin{abstract}
Iterative detection of Diagonal Block Space Time Trellis Codes (DBSTTCs), Trellis Coded Modulation (TCM) and Reversible Variable Length Codes (RVLCs) is proposed. With the aid of efficient iterative decoding, the proposed scheme is capable of providing full transmit diversity and a near channel capacity performance. The performance of the proposed scheme was evaluated when communicating over uncorrelated Rayleigh fading channels. Explicitly, significant iteration gains were achieved by the proposed scheme, which was capable of performing within $2 \mathrm{~dB}$ from the channel capacity.
\end{abstract}

\section{INTRODUCTION}

Delay Diversity Codes (DDCs) [2,3] constitute a subclass of Space Time Trellis Codes (STTCs), which employ a simple spatial repetition code transmitting $N$ delayed copies of each information symbol with the aid of $N$ number of transmit antennas for attaining full transmit diversity. As member of this DDC family, Diagonal Block Space Time Trellis Codes (DBSTTCs) [1, 4] exploit the spatial coding advantage with the aid of a block code, without incurring any extra decoding complexity compared to the original DDC of [2] employing a repetition code. Specifically, each information symbol is first encoded into a codeword of $N$ symbols, then the codeword is transmitted using $N$ number of transmit antennas diagonally across the 'space-time grid' constituted by the antennas and the time-slots used. A block of $L$ number of information symbols is encoded into DBSTTC symbols and the decoding trellis is terminated using $N-1$ number of zero symbols. Hence, the overall coding rate becomes $L /(L+N-1)$, which tends to unity provided that $L$ is sufficiently large with respect to $N$. DBSTTCs have been shown in $[1,4]$ to achieve full transmit diversity with the aid of a minimum number of trellis states while having a coding advantage which is determined by the minimum product distance of the block code employed, when communicating over both quasi-static and uncorrelated or high-Doppler flat Rayleigh fading channels. More explicitly, the required number of trellis states, $S$, is given by $S=2^{b(N-1)}$, where $b=\log _{2}(M)$ is the number of modulated bits per $M$-ary modulated symbol. Note that the STTC employing $N=2$ transmit antennas, having $S=4$ trellis states and using $M=4$-level Phase Shift Keying (4PSK) proposed in [5] is actually a DBSTTC, which achieves a full transmit diversity using the minimum number of trellis states, i.e. at the minimum complexity.

Although the achievable coding advantage of STTCs may be improved by invoking a higher number of trellis states, the extra coding gain obtained is often rather modest in the light of the amount of decoding complexity incurred. Specifically, it has been shown in [6,

The financial support of both the EPSRC, Swindon UK and the EU under the auspices of the Phoenix project is gratefully acknowledged. The authors are also grateful to Dr M. Tao for the discussions regarding [1]. pp. 459-466] that a serially concatenated channel coding and Space Time Block Coding (STBC) [7] scheme, which employs turbo codes and the unity-rate $G_{2}$ STBC of [7] performs better than the corresponding STTC having the same decoding complexity, when a transmit diversity order of $N=2$ was targeted. Therefore, it is beneficial to keep the complexity of the STTC to the minimum, when aiming for full transmit diversity, and to invest the rest of the affordable complexity in a concatenated channel code. The DBSTTC has a unity rate and it is capable of achieving full transmit diversity using the minimum possible number of trellis states, while still benefiting from the coding gain of a block code. Therefore, DBSTTCs constitute attractive schemes for concatenation with channel codes, when aiming for achieving a performance near the channel capacity at full transmit diversity. Furthermore, unlike the decoding of STBC schemes, the detection of DBSTTC is trellis based, hence it can be iteratively turbo-decoded in conjunction with the trellis-based channel decoding scheme employed for the sake of achieving further iteration gains.

Trellis Coded Modulation (TCM) [6, 8] employing a symbolbased interleaver constitutes a bandwidth-efficient joint channel coding and modulation scheme, which was originally designed for transmission over Additive White Gaussian Noise (AWGN) channels. By contrast, Bit-Interleaved Coded Modulation (BICM) [9] employing parallel bit-based interleavers was designed for communicating over uncorrelated Rayleigh fading channels. Therefore, TCM outperforms BICM when communicating over AWGN channels, while the opposite is true when communicating over uncorrelated Rayleigh fading channels. Note that when the transmit diversity order is sufficiently high, the channel's Rayleigh fading envelope is transformed to a Gaussian-like near-constant envelope. Hence, the benefits of a TCM scheme designed for AWGN channels may be efficiently exploited, when TCM is concatenated with DBSTTC.

In most practical scenarios, the source symbols to be transmitted are correlated to a certain degree and hence they are not equiprobable. Lossless Variable Length Codes (VLCs) constitute a family of low-complexity source compression schemes, where the more frequently appearing source symbols are assigned shorter codewords, while the less frequently occurring symbols are assigned longer codewords [10]. In order to exploit the residual redundancy inherent in VLCs, bit-based trellis decoding can be employed. However, VLCs are sensitive to transmission errors, since in case of errors the end of the corrupted VLC cannot be recognised, which may lead to prolonged error propagation. This problem is mitigated to some degree by Reversible VLCs (RVLCs) [11], which were invoked as the outer code in our proposed system. A convolutional code was concatenated with RVLCs as the inner code in the joint source/channel coding scheme of [12]. It was also shown in [13] that RVLCs are amenable to concatenation with TCM for the sake of aiming at a bandwidth and power efficient scheme.

Based on the rationale of our previous arguments, in this con- 


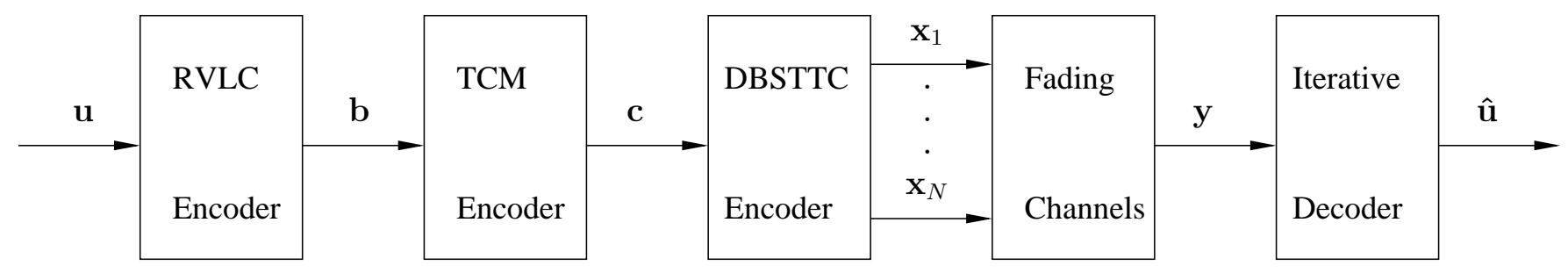

Figure 1: Block diagram of the DBSTTC-TCM-RVLC scheme. The notations $\mathbf{u}, \hat{\mathbf{u}}, \mathbf{b}, \mathbf{x}_{i}$ and $\mathbf{y}$ denote the vectors of the source symbols, the estimates of the source symbols, the RVLC coded bits, the TCM symbols, the DBSTTC coded symbols for transmit antenna $i$ and the received symbols, respectively. The symbol-based channel interleaver between the DBSTTC and TCM schemes as well as the the bit-based interleaver between TCM and RVLC arrangements are not shown for the sake of simplicity.

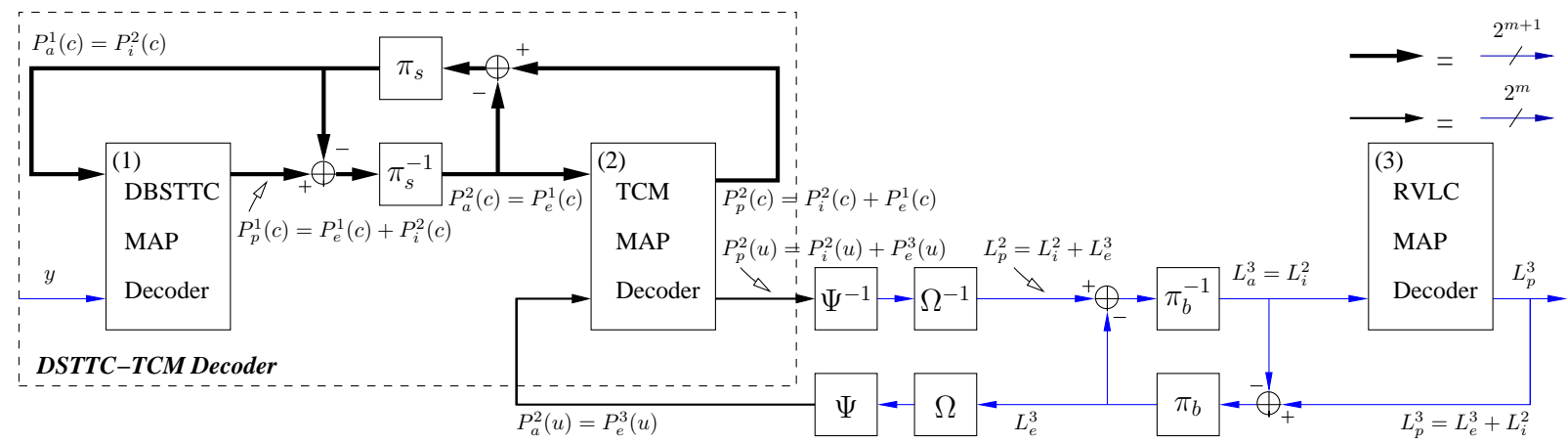

Figure 2: Block diagram of the iterative DBSTTC-TCM-RVLC decoder. The notations $\pi_{(s, b)}$ and $\pi_{(s, b)}^{-1}$ denote the interleaver and deinterleaver, while the subscript $s$ or $b$ denotes the symbol-based or bit-based nature of the interleaver, respectively. Furthermore, $\Psi$ and $\Psi^{-1}$ denote LLRto-symbol and symbol-to-LLR probability conversion, while $\Omega$ and $\Omega^{-1}$ denote the addition and deletion of the LLRs of the side information and dummy bits of the RVLC.

tribution, a jointly optimised space time trellis coded modulation and source-coding scheme based on serially concatenated DBSTTC, TCM and RVLC is proposed. An efficient iterative turbo-detection scheme is utilised for exchanging information between the constituent codes for the sake of achieving full transmit diversity and a near-channelcapacity performance.

\section{SYSTEM OVERVIEW}

We employ the RVLCs designed in [11], where the codewords are $C=\{00,11,010,101,0110\}$ associated with the source symbol sequence of $u=\{0,1,2,3,4\}$. The associated source entropy is 2.14 bits/symbol (BPS) and the average codeword length is 2.46 bits, giving an RVLC coding rate of $R_{R V L C}=2.14 / 2.46=0.87$. The RVLC outer encoder of Figure 1 maps the source symbol sequence $\mathbf{u}$ to a variable-length codeword sequence $\mathbf{b}$, which can be represented as a binary bit sequence at each encoding instance. A minimum number of zero-valued dummy bits are concatenated to the RVLC's output bit sequence, such that we have a constant-length TCM encoder's input sequence. The side information related to the number of RVLC output bits per transmission frame conveying the RVLCs is explicitly signalled to the decoder by repeating the side-information bits three times for the sake of invoking majority logic based detection and then the side information is further protected by the TCM scheme. More explicitly, the resultant bit sequence representing the RVLC output bits, dummy bits and side information bits is then treated as the input of the TCM encoder of Figure 1, which has a coding rate of $R_{c m}=\frac{m}{m+1}$ and employs a $2^{m+1}$-level modulation scheme [6].

The structure of the novel DBSTTC-TCM-RVLC turbo-detection scheme is illustrated in Figure 2, where there are three constituent decoders, each labelled with a round-bracketed index. Two symbolbased and one bit-based MAP decoders [6] were employed, each operating in the logarithmic-domain, for the sake of decoding the DBSTTC, TCM and RVLC, respectively. The notations $P(c)$ and $P(u)$ denote the symbol probabilities of the $(m+1)$ coded bits and the $m$ uncoded information bits of the TCM scheme, respectively. The subscripts of $p, e, a$ and $i$ denote the a posteriori, extrinsic, a priori and intrinsic nature of the corresponding symbol probability, respectively. The notations $L_{p}, L_{e}$ and $L_{i}$ denote the LogarithmicLikelihood Ratio (LLR) of the a posteriori, extrinsic and intrinsic information, respectively. The probabilities as well as the LLRs associated with one of the three constituent decoders having a label of $1 \ldots 3$ are differentiated by the superscript of $1 \ldots 3$. Since the TCM scheme employs a systematic convolutional code, we have $P_{p}^{2}(u)=$ $P_{i}^{2}(u)+P_{e}^{3}(u)$, where $P_{i}^{2}(u)$ denotes the intrinsic probabilities of the uncoded information symbols representing the extrinsic information provided by the decoder itself and the systematic information obtained from the systematic part of $P_{e}^{1}(c)$. Similarly, $P_{i}^{2}(c)$ denotes the intrinsic probabilities of the TCM coded symbols representing the extrinsic information provided by the decoder itself and the systematic part of the codewords obtained from $P_{e}^{3}(u)$. Hence, the DBSTTC and RVLC decoders benefit from each other's extrinsic information through the TCM decoder. At the final decoding iteration, the a posteriori information of the RVLC's coded bit, namely $L_{p}^{3}$ of Figure 2 , is fed to a sequence estimator for estimating the RVLC's source symbol sequence denoted as $\hat{\mathbf{u}}$ in Figure 1.

A DBSTTC-BICM-RVLC system may be created by replacing the TCM encoder/decoder with the BICM encoder/decoder as well as substituting the symbol-based interleaver/deinterleaver between the 
TCM and DBSTTC codecs by parallel bit-based interleavers in Figures 1 and 2. Note that the DBSTTC-BICM decoder is reminiscent of the Iteratively-Decoded BICM (BICM-ID) scheme [14], where a softdecision based demodulator was employed by the BICM-ID scheme instead of the DBSTTC. A Set-Partitioning (SP) scheme [15] labelled as signal mapper was utilised by both the TCM and BICM-ID schemes. We also employed a SP labelled signal mapper for the DBSTTCBICM-RVLC scheme, since we found that the Gray-labelled DBSTTCBICM-RVLC was unable to outperform the SP-based DBSTTC-BICMRVLC arrangement in our iterative decoding scheme.

\section{CODE DESIGN FOR DBSTTC}

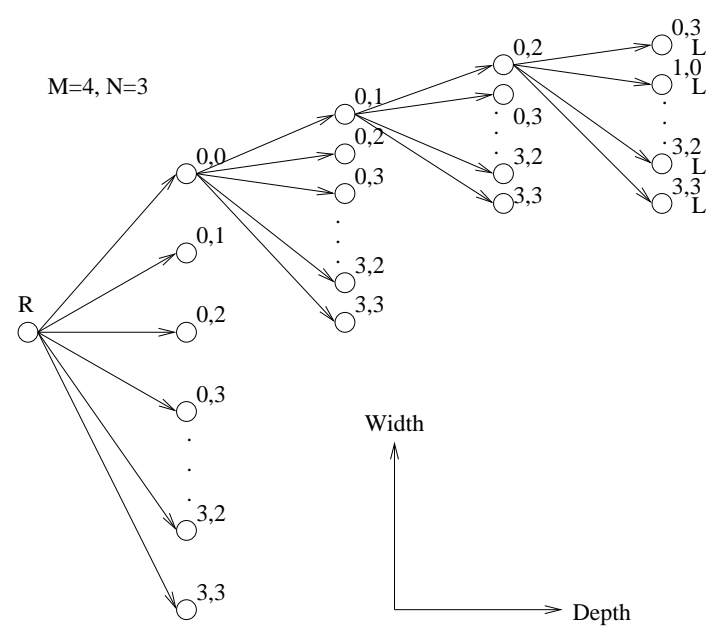

Figure 3: The tree structure of all permutations for DBSTTC employing $N=3$ transmit antennas and an $M=4$-level modulator. The notations $R$ and $L$ denote the root node and leaf nodes, respectively.

A DBSTTC codeword consists of $N$ symbols and the $N$-symbol codeword is transmitted using $N$ number of transmit antennas diagonally allocated across the 'space-time grid' constituted by the antennas and the time-slots used. Therefore, the code design of a DBSTTC can be viewed as finding the best possible permutation for these codewords that give the largest minimum product distance, $P D_{\min }$ [1]. As the transmit antennas are statistically equivalent to each other in the spatial domain, the permutation on the first antenna's transmitted symbols, i.e. the first symbol of an $N$-symbol codeword, can be fixed in the natural order. Hence the permutation search is based on $N-1$ symbols of the $N$-symbol codeword. It was shown in [1] that all the possible permutations for a DBSTTC can be represented by a tree. Figure 3 illustrates the corresponding tree structure of the DBSTTC scheme employing $N=3$ transmit antennas and an $M=4$-level modulator, where each of the permutations ends in a leaf node in the tree. Hence the 'branch-and-bound' algorithm of [16] can be utilised for efficiently solving this permutation based optimisation problem [1]. More specifically, this algorithm forms a tree structure (branching operation), establishes a lower bound for the $P D_{\min }$ (bounding operation) and searches only the specific tree branches that have a local $P D_{\min }$ higher than the lower bound. Figure 4 summarises the operation of this algorithm, where a permutation having a $P D_{\min }$ higher than the lower bound is found, if the current node is found to be a leaf node at block 5 of Figure 4 . Then the lower bound is reinitialised as the newly found $P D_{\min }$ and the process continues, until there are no more branches emerging from the root node.

In this contribution, we studied the effect of modulator choices

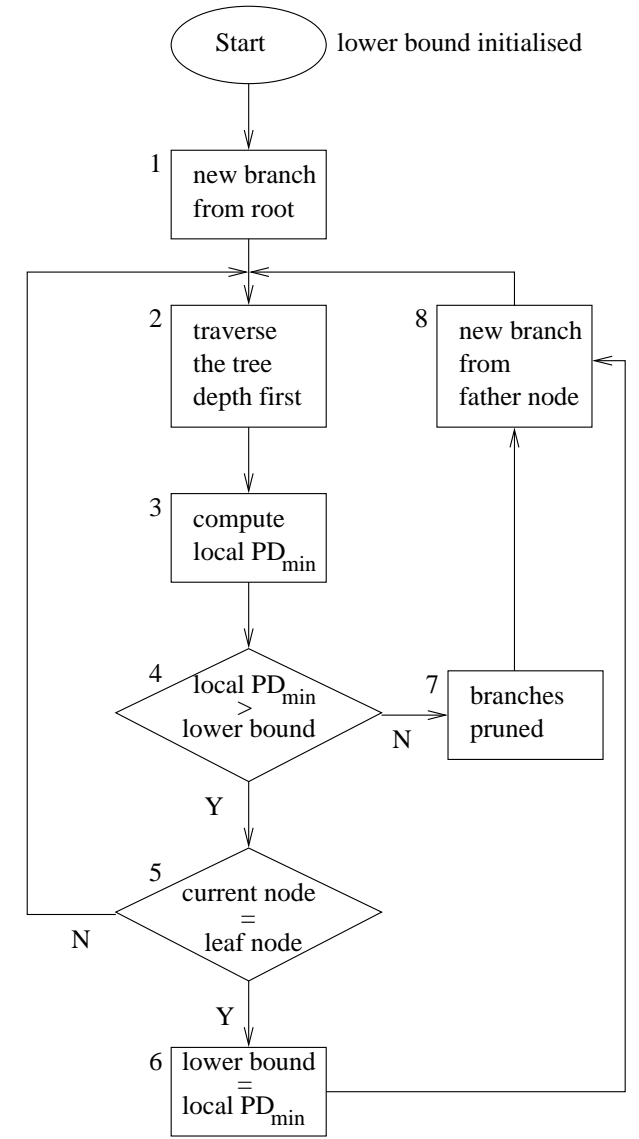

Figure 4: Flow chart of the branch-and-bound algorithm, where the minimum product distance is denoted as $P D_{\min }$.

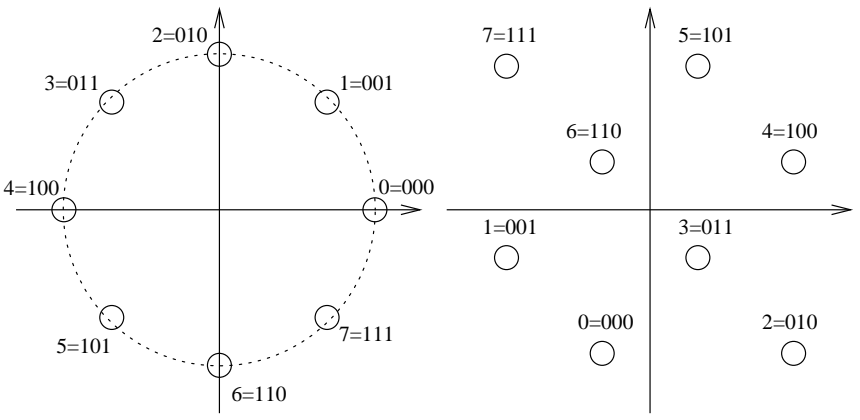

Figure 5: Phasor constellations of 8PSK (left) and 8APSK [8] (right).

on the achievable $P D_{\min }$ using classic 8-level PSK (8PSK), the 8level Amplitude PSK (8APSK) scheme of [8] and a scheme we refer to as $\pi$ /4-rotated 8-level Quadrature Amplitude Modulation ( $\pi / 4$ 8QAM). More explicitly, Figures 5 and 6 depict the phasor constellations of 8PSK, 8APSK and $\pi / 4-8 \mathrm{QAM}$. We found that when we have $\theta=\pi / 4$ and $r_{1} / r_{2}=0.5177$, the corresponding $\theta$-rotated $\theta$-8QAM scheme of Figure 6 gives the best $P D_{\min }$, since we have $E D\left(x_{1}, x_{6}\right)=E D\left(x_{1}, x_{7}\right)=E D\left(x_{6}, x_{7}\right)$, where $x_{i}$ denotes the constellation point assigned to symbol index $i$ and $E D(a, b)$ denotes the Euclidean distance between the constellation points $a$ and $b$. Note that all three modulators are SP-labelled and their SP constructions are identical to that shown in Figure 6. Table 1 summarises the minimum product distance of various DBSTTC schemes using 8PSK, 


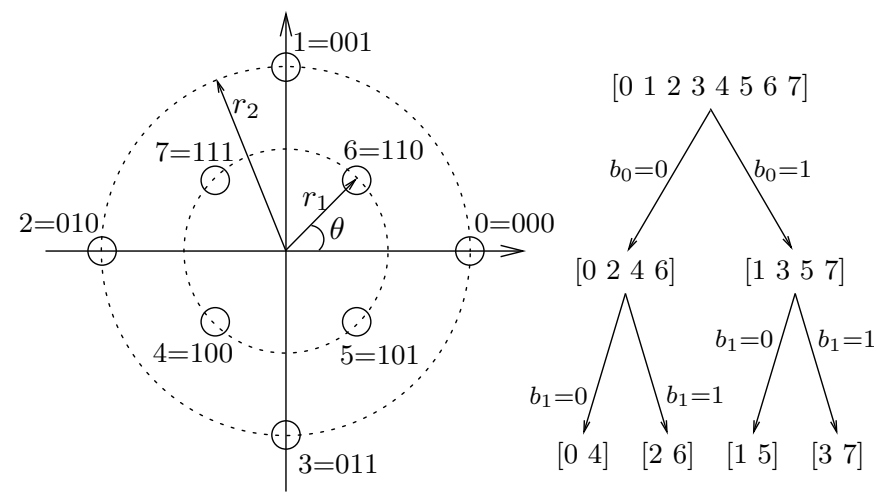

Figure 6: The $\theta$-8QAM modulator (left) having $r_{1} / r_{2}=0.5177$ and $\theta=\pi / 4$ together with its set-partitioning construction (right).

\begin{tabular}{|l|c|c|c|}
\hline$P D_{\min }$ & $8 \mathrm{PSK}$ & $8 \mathrm{APSK}$ & $\pi / 4-8 \mathrm{QAM}$ \\
\hline$N=1$ & 0.59 & 0.80 & $\mathbf{0 . 8 5}$ \\
$N=2$ & 2.00 & 1.28 & $\mathbf{2 . 6 7}$ \\
$N=3$ & $\mathbf{4 . 0 0}$ & 2.56 & 2.25 \\
\hline
\end{tabular}

Table 1: The minimum product distance, $P D_{\text {min }}$, of DBSTTC schemes employing $N$ transmit antennas based on 8PSK, 8APSK and $\pi / 4-8 \mathrm{QAM}$.

8APSK and $\pi / 4-8 \mathrm{QAM}$. Note that when we have $N=1, P D_{\min }$ is simply given by the squared minimum Euclidean distance of the uncoded constellation. As we can see from Table 1, $\pi / 4-8 \mathrm{QAM}$ is optimum, when we have $N=1$ (uncoded) and $N=2$, whereas 8 PSK is optimum for $N=3$, in terms of $P D_{\min }$. Hence, the best achievable $P D_{\min }$ depends on both the modulator type as well as on the number of transmit antennas of the DBSTTC scheme.

In the next section, we will study the attainable performance of 8PSK-based DBSTTC having $N=2$ and $N=3$, as well as the $\pi / 4$ 8QAM-based DBSTTC arrangement having $N=2$ in the context of the joint iteratively decoded system of Section 2 with the aid of a single receive antenna. The new codeset found for $\pi / 4-8 \mathrm{QAM}$-based

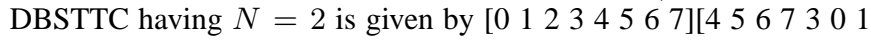
2], while the codeset of 8PSK-based DBSTTC employing $N=2$ and $N=3$ can be found from [1].

\section{SIMULATION RESULTS}

We will evaluate the achievable performance of both DBSTTC-TCMRVLC and DBSTTC-BICM-RVLC using the Bit Error Ratio (BER) versus signal to noise ratio per bit, namely $E_{b} / N_{0}$. The BER is calculated based on the hard decision value of $L_{p}^{3}$ seen in Figure 2 and on the sequence $\mathbf{b}$ of Figure 1. We employed the 64-state TCM scheme of [8] as well as a BICM scheme using Paaske's 64-state nonsystematic convolutional code described for example in [17, p. 331]. We refer to the iteration between the DBSTTC decoder and the TCM/ BICM decoder as an inner iteration $I_{i}$ as well as the iteration between the DBSTTC-TCM/BICM decoder and the RVLC decoder as an outer iteration $I_{o}$. The total number of inner iterations invoked equals to $I_{i}^{t}=I_{o} I_{i}$. The number of TCM/BICM coded symbols per transmission frame was fixed to 1024 8PSK or $\pi / 4-8 \mathrm{QAM}$ symbols and the overall coding rate was $R_{a}=0.5733$. The effective throughput was $\eta=R_{a} \log _{2}(8)=1.72 \mathrm{BPS}$.

Figure 7 shows the BER versus $E_{b} / N_{0}$ performance of DBSTTCTCM/BICM-RVLC employing $N=2$ transmit antennas using both

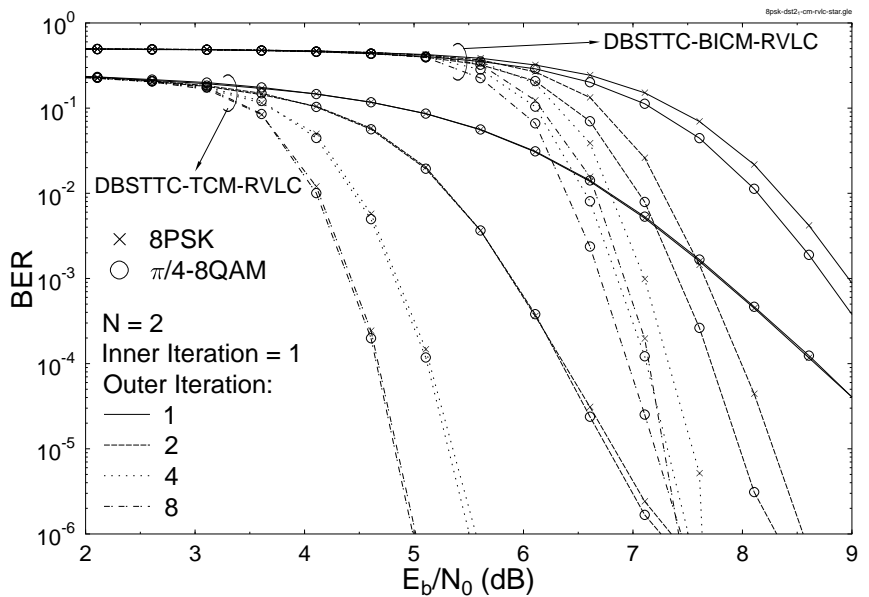

Figure 7: BER versus $E_{b} / N_{0}$ performance of the proposed DBSTTCTCM-RVLC and DBSTTC-BICM-RVLC schemes when communicating over fast Rayleigh fading channels. The effective throughput is 1.72 BPS. and the number of transmit antennas is $N=2$.

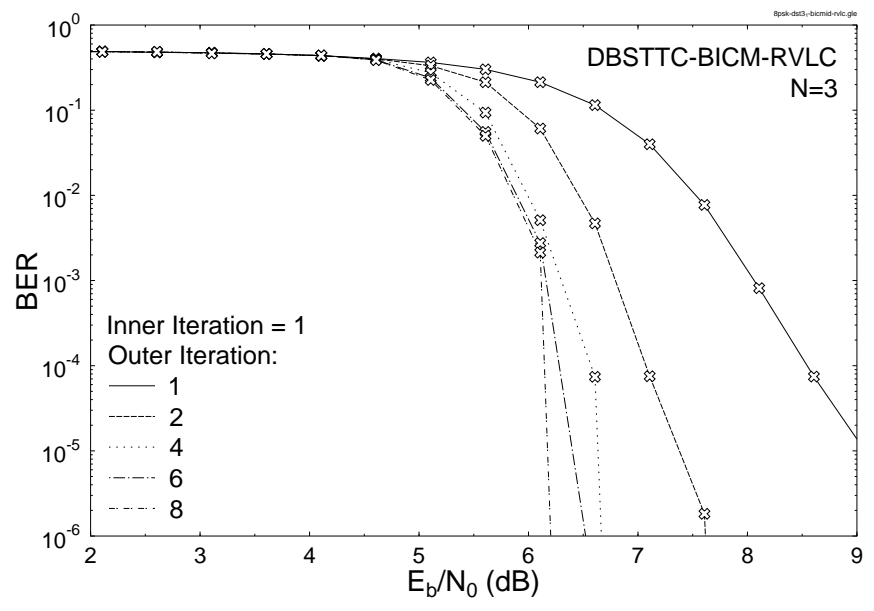

Figure 8: BER versus $E_{b} / N_{0}$ performance of the proposed 8PSKbased DBSTTC-BICM-RVLC scheme when communicating over fast Rayleigh fading channels. The effective throughput is $\mathbf{1 . 7 2}$ BPS. and the number of transmit antennas is $N=3$.

$8 \mathrm{PSK}$ and $\pi / 4-8 \mathrm{QAM}$. We can see from Figure 7 that as predicted, DBSTTC-TCM-RVLC outperformed DBSTTC-BICM-RVLC. However, the advantage of $\pi / 4-8 \mathrm{QAM}$ over 8PSK becomes significant only for DBSTTC-BICM-RVLC, but not for DBSTTC-TCM-RVLC. Figures 8 and 9 illustrate the BER versus $E_{b} / N_{0}$ performance of DBSTTC-TCM/BICM-RVLC employing $N=3$ transmit antennas using 8PSK. It is clear from Figures 7 to 9 that DBSTTC-TCM/BICMRVLC benefits from having both a transmit diversity gain and a significant iteration gain, when the number of transmit antennas is increased. Again, DBSTTC-TCM-RVLC outperformed DBSTTC-BICMRVLC at $N=3$.

As illustrated in Figure 9, a significant iteration gain was achieved, when the number of outer iterations was increased. Explicitly, at $I_{i}=1$ and $I_{o}=1$ there is no feedback from the TCM scheme to the DBSTTC arrangement or from the RVLC to the TCM decoder. When $I_{i}$ was increased from 1 to 4 and we had $I_{o}=1$, iterative decoding exchanging information between the DBSTTC and TCM schemes occurred, but this time there was no feedback from the RVLC 


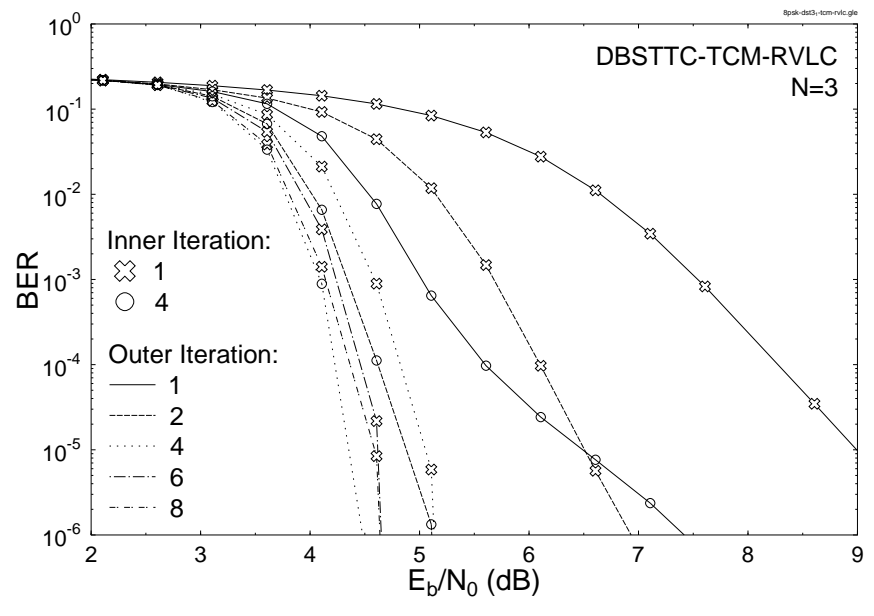

Figure 9: BER versus $E_{b} / N_{0}$ performance of the proposed 8PSKbased DBSTTC-TCM-RVLC scheme when communicating over fast Rayleigh fading channels. The effective throughput is $\mathbf{1 . 7 2}$ BPS. and the number of transmit antennas is $N=3$.

to the TCM decoder yet. It is shown in Figure 9 that at BER $=10^{-4}$ an SNR gain of approximately $2.5 \mathrm{~dB}$ was attained by the scheme having $I_{i}^{t}=I_{i}=4$ and $I_{o}=1$ compared to the non-iterative scheme having $I_{i}^{t}=I_{i}=I_{o}=1$. This shows that the iterative decoding exchanging information between the DBSTTC and TCM schemes was very efficient. Let us now consider the scheme having $I_{i}=1$ and $I_{o}=4$, resulting in a total of $I_{i}^{t}=4$ iterations between the DBSTTC and TCM schemes as well as $I_{o}=4$ iterations between the RVLC and the DBSTTC-TCM decoder. By comparing the BER performance of the scheme having $I_{i}=1$ and $I_{o}=4\left(I_{i}^{t}=4\right)$ to that of the scheme having $I_{i}=4$ and $I_{o}=1\left(I_{i}^{t}=4\right)$ at $\mathrm{BER}=10^{-4}$ in Figure 9, we observe that a further $0.8 \mathrm{~dB}$ SNR gain was achieved, when the the number of iterations $I_{o}$ was increased from 1 to 4 , while having $I_{i}^{t}=4$. Therefore, the iterative decoder exchanging information between the RVLC and DBSTTC-TCM decoders is capable of further enhancing the system's achievable performance.

As we observe from Figures 7 and 9, the best DBSTTC-TCMRVLC scheme using 8PSK requires $E_{b} / N_{0}=4.67 \mathrm{~dB}$ and $E_{b} / N_{0}=$ $4.22 \mathrm{~dB}$ when we have $N=2$ and $N=3$, respectively, for attaining a BER of $10^{-4}$. Note that for an effective throughput of $1.72 \mathrm{BPS}$, the Rayleigh fading channel capacity of an 8PSK-based spacetime code employing $N=2$ and $N=3$ transmit antennas is $E_{b} / N_{0}=2.95 \mathrm{~dB}$ and $E_{b} / N_{0}=2.60 \mathrm{~dB}$, respectively, according to the calculations provided in [18]. Hence the proposed DBSTTC-TCM-RVLC system employing two and three transmit antennas is capable of operating within about $1.72 \mathrm{~dB}$ and $1.62 \mathrm{~dB}$ from the corresponding channel capacity, respectively, when aiming for a throughput of 1.72 BPS using 8PSK.

\section{CONCLUSIONS}

In this contribution the novel concept of amalgamated DBSTTC, TCM/ BICM and RVLC aided transmission was proposed. The achievable performance benefits were demonstrated in the context of the novel iterative turbo-decoding mechanism exchanging information between the constituent decoders, when communicating over uncorrelated Rayleigh fading channels. Specifically, the proposed DBSTTC-TCMRVLC employing two and three transmit antennas was capable of performing within $1.72 \mathrm{~dB}$ and $1.62 \mathrm{~dB}$, respectively, from the cor- responding channel capacity. It was also shown in Section 3 that the optimum DBSTTC design depends on both the modulator type as well as on the number of transmit antennas employed.

\section{REFERENCES}

[1] M. Tao and R. S. Cheng, "Diagonal Block Space-time Code Design for Diversity and Coding Advantage over Flat Rayleigh Fading Channels," IEEE Transactions on Signal Processing, pp. 1012-1020, April 2004.

[2] N. Seshadri and J. H. Winters, "Two signaling schemes for improving the error performance of frequency-division-duplex (FDD) transmission systems using transmit antenna diversity," Int. J. Wireless Information Network, vol. 1, no. 1, pp. 49-60, 1994.

[3] A. Wittneben, "A New Bandwidth Efficient Transmit Antenna Modulation Diversity Scheme for Linear Digital Modulation," International Conference on Communications (ICC), vol. 3, pp. 1630-1634, May 1993.

[4] S. Li, X. Tao, W. Wang, P. Zhang and C. Han, "Generalized Delay Diversity Code: A Simple and Powerful Space-time Coding Scheme," IEEE International Conference on Communication Technology (ICCT), pp. 1697-1703, August 2000.

[5] V. Tarokh, N. Seshadri and A. R. Calderbank, "Space-time Codes for High Rate Wireless Communication: Performance analysis and code construction," IEEE Transactions on Information Theory, vol. 44, pp. 744-765, March 1998.

[6] L. Hanzo, T. H. Liew and B. L. Yeap, Turbo Coding, Turbo Equalisation and Space Time Coding for Transmission over Wireless channels. New York, USA: John Wiley IEEE Press, 2002.

[7] S. M. Alamouti, "A Simple Transmitter Diversity Scheme for Wireless Communications," IEEE Journal on Selected Areas in Communications, vol. 16, pp. 1451-1458, October 1998.

[8] G. Ungerböck, "Channel Coding with Multilevel/Phase Signals," IEEE Transactions on Information Theory, vol. 28, pp. 55-67, January 1982.

[9] E. Zehavi, "8-PSK trellis codes for a Rayleigh fading channel," IEEE Transactions on Communications, vol. 40, pp. 873-883, May 1992.

[10] L. Hanzo, P.J. Cherriman and J. Street, Wireless Video Communications: Second to Third Generation Systems and Beyond. NJ, USA : IEEE Press., 2001

[11] Y. Takishima, M. Wada and H. Murakami, "Reversible Variable Length Codes," IEEE Transactions on Communications, vol. COM-43, no. 2/3/4, pp. 158-162, 1995.

[12] R. Bauer, J. Hagenauer, "On Variable Length Codes for Iterative Source/Channel Decoding," in IEEE Data Compression Conference, (UT, USA), pp. 273-282, 27-29 March 2001.

[13] S. X. Ng, F. Guo, J. Wang, L-L. Yang and L. Hanzo, "Joint Sourcecoding, Channel-coding and Modulation schemes for AWGN and Rayleigh Fading Channels," IEE Electronics Letters, vol. 39, pp. 1259-1261, August 2003.

[14] X. Li, J. A. Ritcey, "Bit-interleaved coded modulation with iterative decoding using soft feedback," IEE Electronics Letters, vol. 34, pp. 942943, May 1998.

[15] D. Divsalar and M. K. Simon, "The design of trellis coded MPSK for fading channel: Set partitioning for optimum code design," IEEE Transactions on Communications, vol. 36, pp. 1013-1021, September 1988.

[16] G. L. Nemhauser, A. H. G. Rinnooy Kan and M. J. Todd, Optimization. Amsterdam, The Netherlands: North-Holland, 1989.

[17] S. Lin and D. J. Costello, Jr, Error Control Coding: Fundamentals and Applications. Inc. Englewood Cliffs, New Jersey 07632: Prentice-Hall, 1983.

[18] S. X. Ng and L. Hanzo, "On the MIMO Channel Capacity of MultiDimensional Signal Sets," in IEEE Vehicular Technology Conference, (Los Angeles, USA), 26-29 September 2004. 